\title{
Prothrombinkomplex-Konzentrat wirksamer als Fresh Frozen Plasma
}

Fragestellung: Ist bei Patienten mit durch Vitamin-K-Antagonisten (VKA) induzierten intrakraniellen Blutungen zur Therapie Prothrombinkomplex-Konzentrat (PCC) oder Fresh Frozen Plasma (FFP) besser wirksam?

Hintergrund: Die VKA-induzierte intrakranielle Blutung ist die gefürchtetste Komplikation einer oralen Antikoagulation bei Patienten mit Vorhofflimmern, tiefen Beinvenenthrombosen oder Lungenembolien. Die Inzidenz von VKA-induzierten intraraniellen Blutungen liegt zwischen $0,3 \%$ und $1 \%$ pro Jahr. Die Sterblichkeit beträgt 30 - 50\%, wobei der wichtigste Prädiktor für eine erhöhte Sterblichkeit die Größenzunahme der initialen Blutung ist. Prinzipiell gibt es mit PCC und FFP zwei Möglichkeiten, eine solche Blutung zu behandeln. Eine Operation verbietet sich in den meisten Fällen, da eine signifikante Störung der Gerinnung vorliegt. Retrospektive Registerdaten hatten in der Vergangenheit nahegelegt, dass es bezüglich des Endpunktes keinen Unterschied macht, ob Patienten mit PCC oder FFP behandelt werden. Die INCH-Studie untersuchte erstmals die Wirksamkeit der beiden Therapieansätze in einem prospektiven randomisierten Design.

Steiner T, Poli S, Griebe M et al. Fresh frozen plasma versus prothrombin complex concentrate in patients with intracranial haemorrhage related to vitamin $\mathrm{K}$ antagonists (INCH): a randomised trial. Lancet Neurol 2016; 15: $566-73$
Patienten und Methodik: Es handelte sich um eine Investigator-initiierte, multizentrische, prospektive, randomisierte, offene Studie mit verblindeter Adjudizierung der Endpunkte. Eingeschlossen wurden Patienten mit intrakraniellen Blutungen un- ter einer VKA-Therapie, die sich innerhalb von zwei Stunden nach Symptombeginn in der Klinik vorstellten, und bei denen die INR 2,0 oder größer war. Die Patienten erhielten entweder $20 \mathrm{ml} / \mathrm{kg}$ FFP oder $30 \mathrm{IE} / \mathrm{kg}$ i.v. PCC innerhalb von einer Stunde nach dem initialen CT. Der primäre Endpunkt war die Zahl der Patienten mit einer INR (International Normalized Ratio) von 1,2 oder niedriger innerhalb von drei Stunden nach Beginn der Therapie.

Ergebnisse: Zwischen August 2009 und Januar 2015 wurden 54 Patienten in die Studie eingeschlossen, von denen 26 FFP und 28 PCC erhielten. Zwei von 23 Patienten (9\%) der FFP-Gruppe erreichten den primären Endpunkt verglichen mit 18 von 27 (67\%) der PCC-Gruppe. Dieser Unterschied war mit einer Odds Ratio von 30,6 statistisch signifikant. 13 Patienten starben, davon acht in der FFP-Gruppe und fünf in der PCC-Gruppe. Innerhalb der ersten drei Tage traten drei thromboembolische Komplikationen auf und innerhalb von zwölf Tagen sechs. Die Zahl unerwünschter Ereignisse war in beiden Gruppen gleich häufig. Das CT nach drei Stunden zeigte eine Zunahme des Hämatoms um $24 \mathrm{ml}$ in der FFP-Gruppe und um 9,7 $\mathrm{ml}$ in der PCC-Gruppe. Nach 24 Stunden waren die entsprechenden Zahlen $22 \mathrm{ml}$ und $8 \mathrm{ml}$. Eine Größenzunahme von mehr als $33 \%$ oder Tod wurde bei $60 \%$ in der FFP-Gruppe beobachtet und bei $30 \%$ in der PCC-Gruppe. Dieser Unterschied war statistisch signifikant.

Schlussfolgerungen: Bei Patienten mit intrakraniellen Blutungen im Rahmen einer Antikoagulation mit VKA scheint PCC wirksamer als FFP zu sein. Dies bezieht sich auf die Normalisierung der INR sowie auf die Größenzunahme des initialen Hämatoms.

\section{PCC ist Therapie der Wahl bei VKA-induzierter intrakranieller Blutung}

Diese in Deutschland durchgeführte Untersuchung ist die erste randomisierte prospektive Studie, welche die beiden Therapieansätze FFP und PCC bei Patienten mit intrakraniellen Blutungen unter VKA in einem randomisierten Design untersuchte. Die Rekrutierung in die Studie war außerordentlich schwierig. Dies erklärt, warum letztendlich in sechs Jahren nur 50 Patienten tatsächlich behandelt wurden.

Die Anzahl der behandelten Patienten war viel zu gering, um Aussagen darüber treffen zu können, ob die beiden Therapien sich in ihrer Wirksamkeit bezüglich klinischer Endpunkte unterscheiden. Bezogen auf die Surrogatparameter Normalisierung der INR, Zeit bis zur Normalisierung der INR und Größenzunahme des initialen Hämatoms war die Behandlung mit PCC allerdings der Gabe von FFP eindeutig überlegen. FFP hat darüber hinaus das Problem, dass es bei Patienten mit Herzinsuffizienz nicht eingesetzt werden kann.

Im Gegensatz zu dem neuen spezifischen Gegenmittel gegen Thrombininhibitoren und Faktor-Xa-Antagonisten haben PCC und FFP auch prothrombotische Eigenschaften, die in ischämischen Insulten und Lungenembolien resultieren können.

Bezogen auf den derzeitigen Therapiestandard sollte PCC als Behandlung der ersten Wahl bei Phenprocoumon-induzierten intrakraniellen Blutungen eingesetzt werden. Für die neuen Antikoagulanzien kommt schon jetzt Idarucizumab für Dabigatran zum Einsatz, während die Zulassung von Andexanet als Gegenmittel für die Faktor-Xa-Hemmer zurzeit noch aussteht. 